\title{
Improvement of Graphene FET Characteristics by Eliminating Aromatic Rings in Fabrication Resist
}

\author{
Hiroko Nakamura* and Reiko Yoshimura \\ Corporate Research \& Development Center, Toshiba Corporation, \\ 1, Komukai Toshiba-cho, Saiwai-ku, Kawasaki 212-8583, Japan \\ *hiroko2.nakamura@toshiba.co.jp
}

\begin{abstract}
Graphene is known to have various specific properties, so that many types of graphene devices are proposed and fabricated. Novolac resist is commonly used to fabricate graphene sensors. Because graphene is composed of six-membered rings of carbon atoms, graphene is thought to be contaminated with the novolac resist by $\pi-\pi$ stacking between graphene and the benzene rings of the novolac resin contained in the novolac resist. The transfer characteristics of graphene field effect transistors (FETs) were used to evaluate the graphene deterioration by fabrication resist. Decreasing the number of novolac resist processes on graphene was effective in suppressing the deterioration of graphene. To avoid the $\pi-\pi$ stacking between graphene and the aromatic rings, the novolac resist was changed to the acryl resist, the base resin of which does not contain aromatic rings. The PAG that has a steric hindrance from approaching a graphene sheet was effective in suppressing the deterioration compared with the PAG without the steric hindrance. These measures suppressed the deterioration of graphene and improved the transfer characteristics of graphene FETs.
\end{abstract}

Keywords: Graphene, Novolac resist, Acryl resist, Graphene FET, Steric hindrance

\section{Introduction}

Graphene is known to have specific properties because of two-dimensional material [1]. Many applications have been proposed, such as high radio frequency transistors [2], sensors [3-5], and photonic devices $[6,7]$ etc. Developing the formation methods of chemical vapor deposition (CVD) graphene [8-10] expanded the range of possibility for graphene devices due to the ease of applying semiconductor processes to the graphene device fabrication. However, there have been discrepancies between the simulation results of the ideal graphene and the experimental results. It is necessary to improve the performance of actual devices close to that of ideal one.

One reason for the low performance of fabricated graphene devices is thought to be the contamination of graphene during the device fabrication. Polymethylmethacrylate (PMMA) resist has been used to fabricate graphene devices by the electron beam (EB) lithography. The effects of PMMA resist and cleaning methods were reported previously $[11,12]$. However, the throughput of EB lithography with PMMA resist is low, which result in a high cost. Novolac resist [13] is often used for sensor fabrication because the necessary pattern sizes can be formed and high throughput can be obtained by the use of the ultraviolet (UV) lithography. Novolac resist is composed of novolac resin, as shown in Fig. 1 (a), and photoactive compound, diazonaphthoquinone, as shown in Fig. 1 (b), both of which contain benzene rings. Graphene is composed of sixmembered rings of carbon atoms, so that the $\pi-\pi$ stacking is suspected between graphene and the benzene ring as shown in Fig. 2. (a)

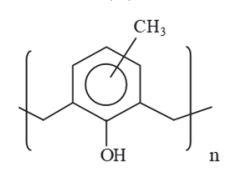

(b)

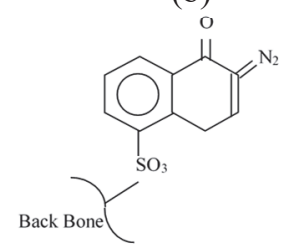

Fig. 1. Main components of novolac resist. (a) Novolac resin, (b) photoactive compound, diazonaphthoquinone. 


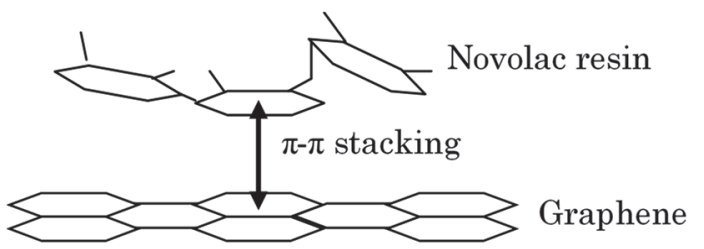

Fig. 2. Deterioration of graphene by $\pi-\pi$ stacking with novolac resin.

There are other types of resist. Argon fluoride (ArF) resist is designed not to contain aromatic rings in order to avoid absorbing ArF excimer laser light [14-16]. Figure 3 shows one example of the ArF resist [16]. The base resin of ArF resist is acrylic or methacrylic resins. Lactone and cycloolefin groups, such as adamantyl groups shown in Fig. 3, are used mainly as the protective groups. It is inferred that the resin dose not stick to a graphene surface.

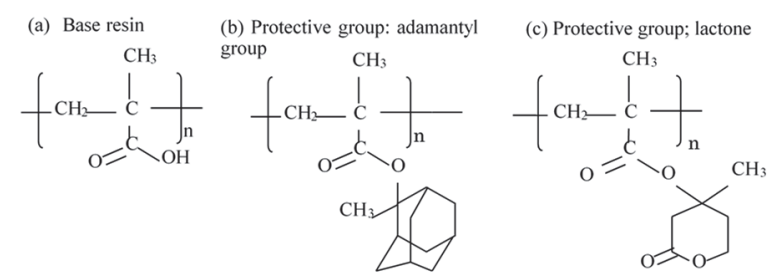

Fig. 3. Example of ArF resist [16].

In this paper, the effects of novolac resist processes on graphene is first examined by the transfer curves of graphene field effect transistors (FETs). Then, the suppression of the deterioration in graphene was investigated using a mixture of $\mathrm{ArF}$ resist and a photoacid generator (PAG) that has a steric hindrance from approaching graphene surfaces.

\section{Experimental}

Commercially available graphene segregated on copper $(\mathrm{Cu})$ sheets was used to fabricate graphene FETs.

Graphene transfer was performed as follows. First, PMMA was coated on a graphene sheet. $\mathrm{A} \mathrm{Cu}$ sheet was dissolved in an ammonium peroxodisulfate solution. The graphene sheet was scooped with a substrate. Two types of substrates were used. One was $285-\mathrm{nm}$-thick thermal silicon oxide $\left(\mathrm{SiO}_{2}\right)$ film deposited on n-type degenerated $\mathrm{Si}$, the backside of which was coated with metal $(100 \mathrm{~nm} \mathrm{Ag} / 20 \mathrm{~nm} \mathrm{Ti})$ as a backside gate electrode. The other type of substrates was the processed one of the first substrate. Drain and source electrodes made of metal $(20 \mathrm{~nm} \mathrm{Au} / 10 \mathrm{~nm} \mathrm{Ni})$ were fabricated on the $\mathrm{SiO}_{2}$ film of the first substrate.
PMMA was removed with acetone. The transferred graphene was annealed at $300{ }^{\circ} \mathrm{C}$ in the mixture of $3 \%$ hydrogen $\left(\mathrm{H}_{2}\right)$ and $97 \%$ argon $(\mathrm{Ar})$ gases.

The source and drain electrodes were formed by a lift-off process. Eaves-shapes were formed by an alkali-soluble film and novolac resist patterns. The alkali-soluble film and the novolac resist were available commercially.

Trimmed graphene patterns were fabricated using three types of resist exposed by a contact aligner, the light source of which was a mercury lamp. One type of resist is commercial novolac resist. The other types of resist were the mixtures of commercial ArF resist and a PAG sensitive to a mercury lamp. The resist is referred to as acryl resist below. Two types of the acryl resist were made by adding different types of PAGs. The PAGs, namely, NDS-105 and NAI-105, were purchased from Midori Kagaku Co., Ltd. $0.2 \mathrm{wt} \%$ of the PAGs were added to the ArF resist. The acryl resist was baked at $130{ }^{\circ} \mathrm{C}$ for $90 \mathrm{sec}$ after exposure of the light. The resist was developed with a tetramethylammonium hydroxide (TMAH) solution.

The excess graphene was etched by oxygen reactive ion etching with the resist pattern as a mask. The resist was removed with $\mathrm{N}$-methyl-2pyrrolidone (NMP).

Before electrical measurements, a graphene FET was annealed at $300{ }^{\circ} \mathrm{C}$ in the mixture of $3 \%$ $\mathrm{H}_{2}$ and $97 \%$ Ar gases.

The transfer curves of graphene FETs were measured by B1500A semiconductor parameter analyzer supplied by Keysight technologies. Drain voltage was $0.1 \mathrm{~V}$. Channel length was $5 \mu \mathrm{m}$ and channel width was $10 \mu \mathrm{m}$.

Raman spectra was measured using LabRAM HR800 Raman spectrometer supplied by HORIBA Jobin Yvon. The wavelength of the excitation light was $523 \mathrm{~nm}$ and the grating was 600 lines $/ \mathrm{mm}$. The wavenumber was calibrated with $520-\mathrm{cm}^{-1}$ Si peak.

The effective mobility of graphene was calculated from the equation below assuming the linear region of a MOSFET [17].

$$
\mu=\frac{L}{W \cdot C_{O X}} \cdot \frac{1}{V} \cdot \frac{d I_{d}}{d V_{g}}
$$

Here, $\mu$ is effective mobility, $L$ is channel length, $W$ is channel width, $V_{\mathrm{d}}$ is drain voltage, $I_{\mathrm{d}}$ is drain current, $V_{\mathrm{g}}$ is gate voltage, and $C_{\mathrm{ox}}$ is capacitance per area.

The stable conformations of PAGs were calculated with the Gaussian09 programs [18] by 
density functional method (B3LYP $[19,20] /$ CEP$31 \mathrm{G}[21])$.

\section{Results and discussion}

3.1. Effects of novolac resist processes on graphene

To confirm the effects of novolac resist processes on graphene devices, FETs were fabricated by two methods. The first method includes two resist processes on graphene as shown in Fig. 4 (a) (two-process method). First, graphene was transferred to a $\mathrm{SiO}_{2}$ substrate. Then, electrodes were fabricated and the graphene sheet was trimmed with novolac resist. The other method includes one resist process on graphene as shown in Fig. 4 (b) (one-process method). Electrodes were fabricated before the graphene transfer to a $\mathrm{SiO}_{2}$ substrate. After graphene transfer, graphene was trimmed with novolac resist. Graphene sheets purchased at the same time were divided into two groups for the two methods. (a)

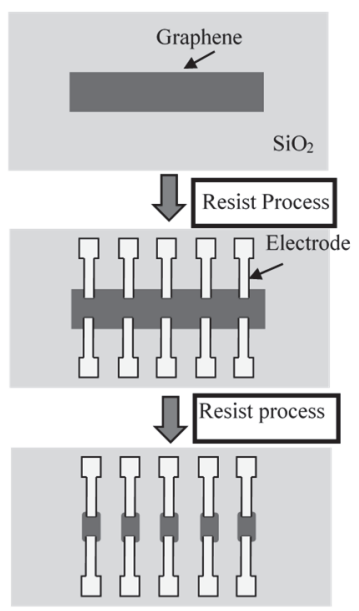

(b)

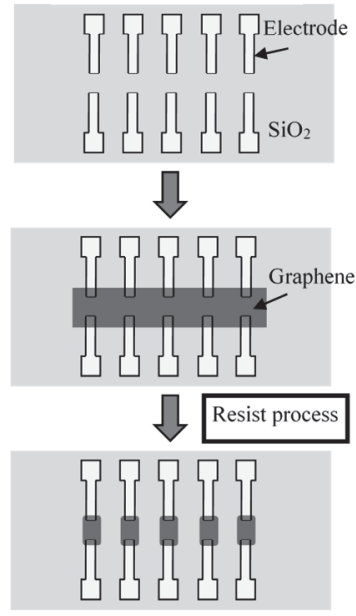

Fig. 4. Graphene FET fabrication methods. (a) Twoprocess method, (b) one-process method.

Figure 5 shows the transfer curves of graphene FETs in one chip. Figure 5 (a) is the case of the twoprocess method. Figure 5 (b) is the case of the oneprocess method. All of the FETs except for those whose electrical connections were obviously short or open are shown. Graphene has two types of carriers, such as electrons and holes. When gate voltage $\left(V_{\mathrm{g}}\right)$ is swept, hole current is measured as drain current $\left(I_{\mathrm{d}}\right)$ in negative voltage and electron current is measured in positive voltage in the neutralization condition [1]. The minimum point of $I_{d}$ is called the Dirac point. When graphene is doped by contaminants, the position of the Dirac point moves $[11,12,22]$. In the case of hole dopants, such as water and resist etc., the Dirac point moves in the positive direction. In Fig. 5 (a), the Dirac points were not observed below $60 \mathrm{~V}$ of $V_{\mathrm{g}}$, which means that the graphene was highly doped during the FET fabrication. On the other hand, the Dirac points were observed around $35 \mathrm{~V}$ of $V_{\mathrm{g}}$ in Fig. 5 (b). Although the graphene subjected to the one-process method was also doped, the degree of doping was less than that of the graphene subjected to the two-process method. Decreasing the number of resist processes on graphene suppressed the deterioration of graphene. Novolac resist processes on graphene are thought to degrade the performance of graphene FETs. (a)

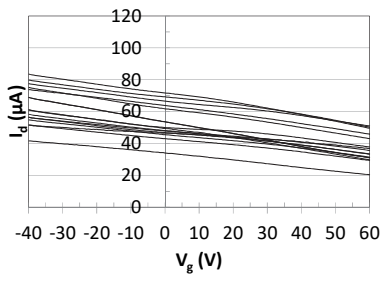

(b)

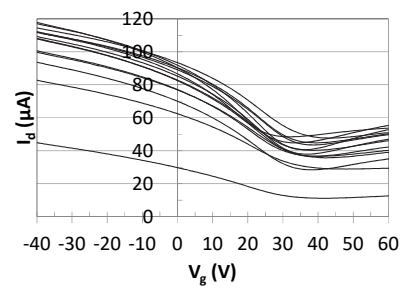

Fig. 5. Transfer curves of graphene FETs fabricated by (a) two-process method and (b) one-process method.
$2 \mathrm{D}$ band

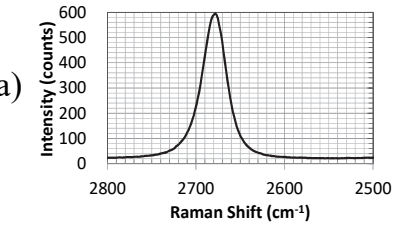

(b)

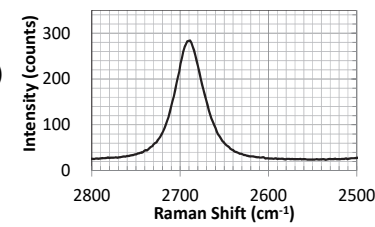

G band
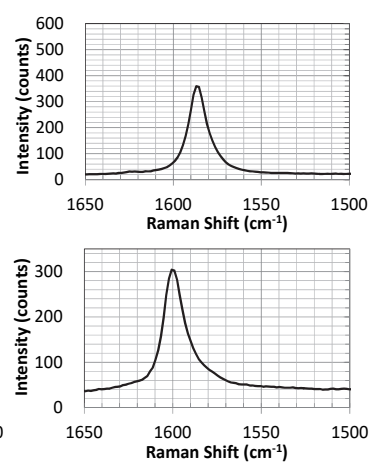

Fig. 6. Raman spectra of graphene in two-process method (a) after graphene transfer and (b) after FET fabrication.
2D band

(a)

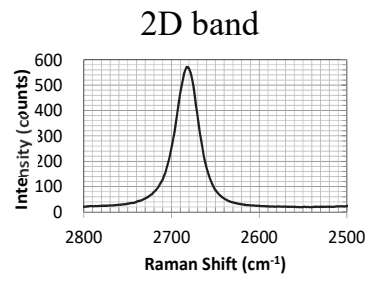

(b)

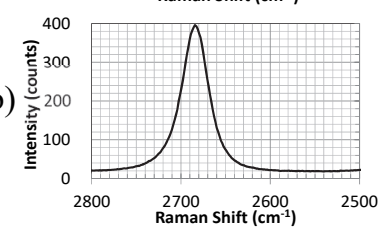

G band
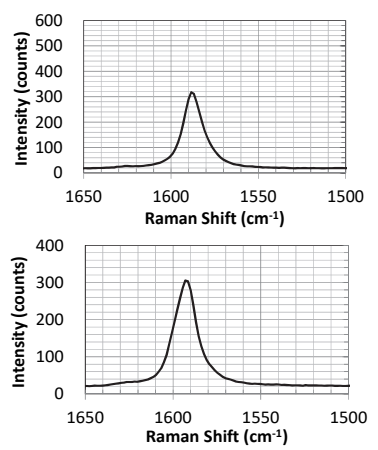

Fig. 7. Raman spectra of graphene in one-process method (a) after graphene transfer and (b) after FET fabrication. 
Figures 6 and 7 show Raman spectra of graphene fabricated by the two-resist process method and by the one-resist process method, respectively, after the graphene transfer to substrates (a) and after the FET fabrication (b). The Raman spectra after the graphene transfer to substrates were nearly the same in both methods. After FET fabrication, the ratio of the 2D band intensity $\left(I_{2 \mathrm{D}}\right)$ to the $\mathrm{G}$ band intensity $\left(I_{\mathrm{G}}\right)$ decreased. The $I_{2 \mathrm{D}} / I_{\mathrm{G}}$ of the two-process method decreased more than that of the one-process method. In addition to that, the peak of the $G$ band was shifted in the two-process method and the 2D band of the two-process method became broader than that of the one-process method.

$I_{2 \mathrm{D}} / I_{\mathrm{G}}$ is sometimes used as an index of graphene deterioration. The $\mathrm{G}$ band is based on the intra band transitions. The 2D band is based on the inter band transitions. When the bands split, there are more transition paths in the 2D band than those in the $\mathrm{G}$ band $[23,24]$. When graphene is contaminated, the bands split, so that $\mathrm{I}_{2 \mathrm{D}} / \mathrm{I}_{\mathrm{G}}$ decreases and the band width of the $2 \mathrm{D}$ band became broad.

The Raman spectra also showed that the novolac resist process deteriorates graphene.

\subsection{Acryl resist}

The Aromatic rings in novolac resin are thought to be the cause of contamination. The aromatic rings stick to graphene surface by $\pi-\pi$ stacking, which deteriorates graphene. ArF resist is designed not to contain aromatic rings in order to avoid absorbing exposure light. Therefore, ArF resist was used instead of novolac resist.

Because the PAGs in commercial ArF resist do not generate acid by the exposure to a mercury lamp, the PAG (NDS-105), which is sensitive to a mercury lamp, was added to the commercial ArF resist. The mixture of the ArF resist and NDS-105 is referred to as acryl resist below.

After graphene transfer (Fig. 4 (b) middle), four chips were divided into two groups. In one group, the commercial novolac resist was used to trim graphene. In the other group, the acryl resist was used. Figure 8 shows the transfer curves of graphene FETs in one chip. Figure 8 (a) is the case of FETs fabricated with the novolac resist and Fig. 8 (b) is the case of FETs fabricated with the acryl resist. All of the FETs except for those whose electrical connections were obviously short or open are shown. The positions of the Dirac points of the FETs fabricated with the acryl resist were slightly lower than those of the FETs fabricated with the novolac resist. The gradients of the transfer curves of the FETs fabricated with the novolac resist were less than those of the FETs fabricated with the acryl resist. To clarify the differences in the gradients of transfer curves between the novolac resist and the acryl resist, the maximum effective mobility was calculated assuming the linear region of a MOSFET as shown in Eq. 1. The effective mobility is proportional to the gradient of an $I_{\mathrm{d}}-V_{\mathrm{g}}$ curve. Figure 9 shows the histograms of the maximum effective mobility according to resist types. The results for two chips of each resist type are shown. Figure 9 (a) is hole mobility and Fig. 9 (b) is electron mobility. The bars filled with diagonal lines represent the case of the acryl resist and the dotted bars represent the case of the novolac resist. In both holes and electrons, mobility of FETs fabricated with the acryl resist was higher than that of FETs fabricated with the novolac resist. Therefore, the acryl resist suppresses the performance degradation in graphene FETs. (a)

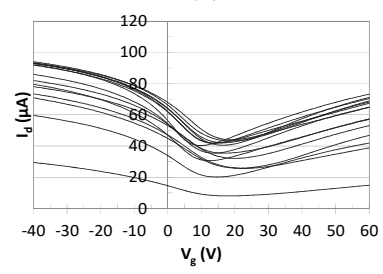

(b)

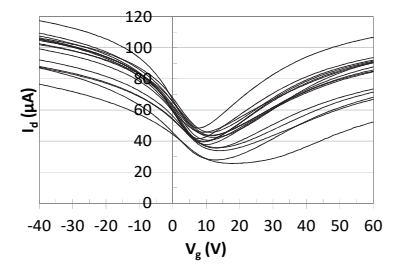

Fig. 8. Transfer curves of graphene FETs fabricated with (a) novolac resist and (b) acryl resist. (a)

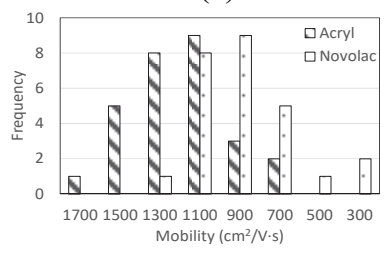

(b)

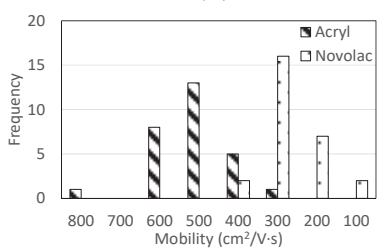

Fig. 9. Mobility histograms according to resin types. Bars filled with diagonal lines represent acryl resist. Dotted bars represent novolac resist. (a) Hole mobility, (b) electron mobility.

The dependence of Raman spectra on types of resist used to trim graphene is shown in Figs. 10 and 11. Figures 10 and 11 are the case of the novolac resist and that of the acryl resist, respectively, after the graphene transfer (a) and after the FET fabrication (b). Initial Raman spectra were nearly the same in both types of resist. In the case of the novolac resist, $I_{2 \mathrm{D}} / I_{\mathrm{G}}$ after $\mathrm{FET}$ fabrication decreased. On the other hand, in the case of the acryl resist, $I_{2 \mathrm{D}} / I_{\mathrm{G}}$ after $\mathrm{FET}$ fabrication was nearly the same as that after graphene transfer. So that, Raman 
spectra also showed that the novolac resist deteriorates graphene more than the acryl resist does.

(a)
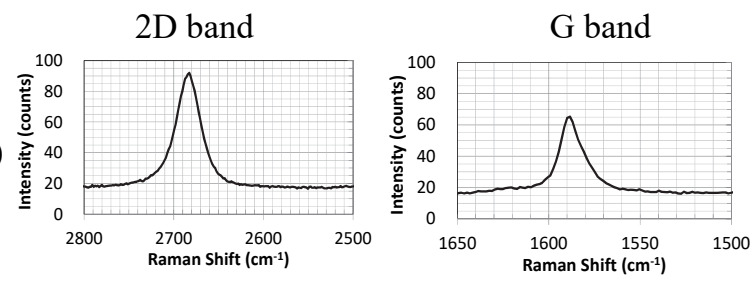

(b)
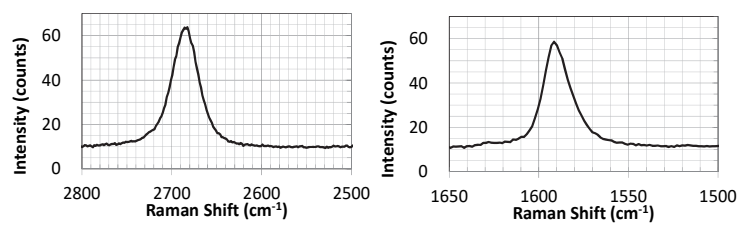

Fig. 10. Raman spectra of graphene using novolac resist (a) after graphene transfer and (b) after FET fabrication.

(a)
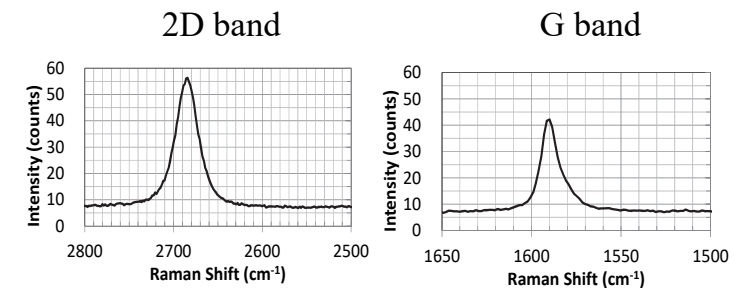

(b)
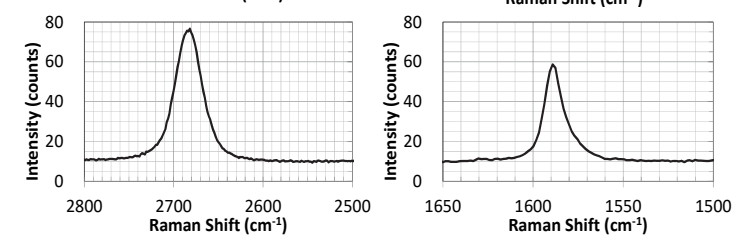

Fig. 11. Raman spectra of graphene using acryl resist (a) after graphene transfer and (b) after FET fabrication.

\subsection{Photoacid generator}

PAGs are indispensable for resist patterning in chemically amplified resist. Because light absorption is necessary to generate acid, a naphthalene ring is necessary in the PAG to absorb the light emitted by a mercury lamp. A steric hindrance of the substituted naphthalene ring contained in the PAG is thought to prevent the naphthalene ring from being parallel to a graphene sheet, which minimizes the sticking of naphthalene rings to graphene. Figure 12 (a) shows the structural formula of NDS-105 used as the PAG difficult to stick to graphene due to a steric hindrance. The calculated stable conformation is shown in Fig. 12 (b). The upper structure is the top view and the bottom one is the side view in Fig. 12 (b). Two methyl groups are stabilized at the upper and lower sides of the planar naphthalene ring. So that, the

bulky group shown as a yellow ellipse in Fig. 12 (c), which corresponds to the part encircled by the red line in Fig. 12 (a), is thought to hinder the naphthalene ring from being parallel to a graphene sheet as shown in Fig. 12 (c). As a result, NDS-105 is unlikely to stick to graphene by $\pi-\pi$ stacking.

(a)

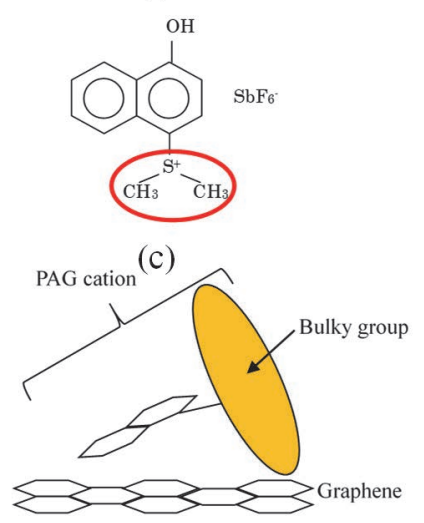

(b)

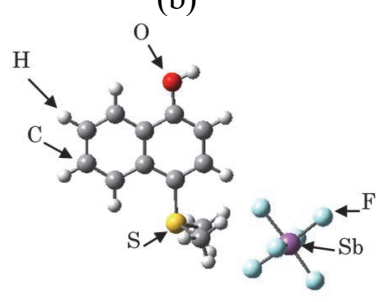

Fig. 12. (a) Structure, (b) stable conformation (top, top view; bottom, side view), and (c) model of interaction with graphene of NDS-105.

In order to compare the effect of the PAG structure, another PAG, the naphthalene ring of which can be parallel to a graphene sheet, was used to trim graphene. Figure 13 (a) shows the structural formula of the PAG, NAI-105. The calculated stable conformation is shown in Fig. 13 (b). The upper structure is the top view and the bottom one is the side view in Fig. 13 (b). Although NAI-105 has a bulky group, which is the yellow circle shown in Fig. 13 (c) and corresponds to the part encircled by the red line in Fig. 13 (a), the bulky group stabilized at one side of the planar naphthalene ring. So that, the naphthalene ring can be parallel to a graphene sheet, which makes it easy to stick to graphene as shown in Fig. 13 (c).

(a)

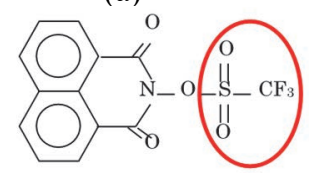

(c)

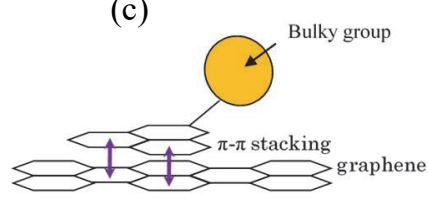

Fig. 13. (a) Structure, (b) stable conformation (top, top view; bottom, side view), and (c) model of interaction with graphene of NAI-105. (b)
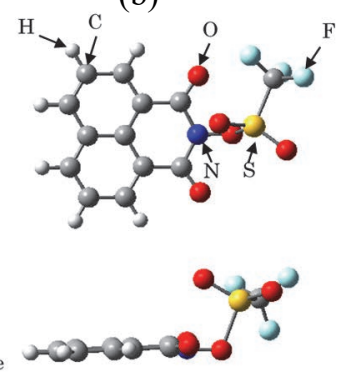
After graphene transfer (Fig. 4 (b) middle), six chips were divided into two groups. In one group, a mixture of commercial ArF resist and NDS-105 was used to trim graphene. In the other group, a mixture of the commercial ArF resist and NAI-105 was used to trim graphene. Figure 14 shows the transfer curves of graphene FETs in one chip. Figure 14 (a) shows the case of NDS-105 and Fig. 14 (b) shows the case of NAI-105. All of the FETs except for those, whose electrical connections were obviously short or open, are shown. The positions of the Dirac points of FETs fabricated with NDS-105 were slightly lower than those of FETs fabricated with NAI-105. The gradients of the transfer curves of FETs fabricated with NAI-105 were less than those of FETs fabricated with NDS-105. To clarify the differences in the gradients of the transfer curves between NDS-105 and NAI-105, the maximum effective mobility was calculated assuming the linear region of a MOSFET. Figure 15 shows the histograms of the maximum mobility according to PAG types. The results for three chips of each PAG type are shown. Figure 15 (a) is the hole mobility and Fig. 15 (b) is the electron mobility. Bars filled with diagonal lines represent the case of NDS-105 and the dotted bars represent the case of NAI- 105 . In both holes and electrons, the mobility of FETs fabricated with NDS-105 was higher than that of FETs fabricated with NAI-105. The PAG difficult to stick to graphene due to the steric hindrance suppresses the performance degradation in graphene FETs. (a)

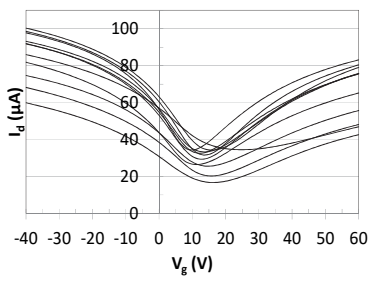

(b)

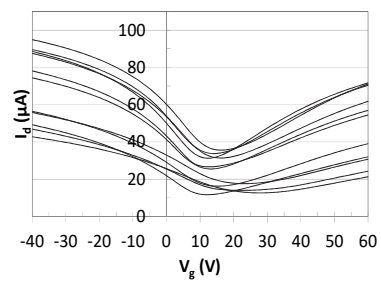

Fig. 14. Transfer curves of graphene FETs fabricated with (a) mixture of ArF resist and NDS-105, and (b) mixture of ArF resist and NAI-105. (a)

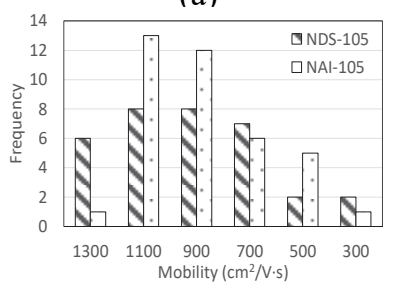

(b)

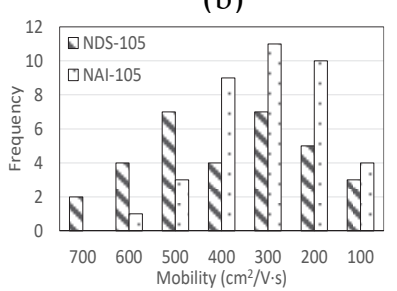

Fig. 15. Mobility histograms according to PAG types. Bars filled with diagonal lines represent NDS-105. Dotted bars represent NAI-105. (a) Hole mobility, (b) electron mobility.
The Raman spectra were nearly same between the PAG types. The distribution of the Raman spectra depending on positions in a graphene sheet was larger than the difference of the Raman spectra between the PAG types.

Commercial ArF resist contains original PAGs that is sensitive to 193-nm ArF excimer laser light. The PAGs may have aromatic rings. So that there is a possibility that the PAGs stick to graphene. If the original PAGs are removed, the degree of deterioration will be reduced.

The experimental conditions of the one-process method in the process number experiment were the same as those of the novolac resist in the resist type experiment. The experimental conditions of the acryl resist in the resist type experiment were the same as those of NDS-105 in the PAG type experiment. However, the results were not the same. One reason is that the graphene quality differed according to production lots. This is the reason why graphene sheets purchased at the same time and chips after graphene transfer at the same time were divided into two groups.

There is the limitation to improve the performance of graphene devices by the acryl resist described above. The usage of the acryl resist only suppresses the deterioration of graphene during graphene trimming. If the initial graphene is of low quality or graphene is damaged during transfer from a $\mathrm{Cu}$ foil to a substrate, the performance of the graphene devices is limited by the initial or damaged quality. The combination of the acryl resist in this paper with high quality graphene is expected to attain high performance of graphene devices.

\section{Conclusion}

The deterioration of graphene during graphene device fabrication was investigated from the viewpoint of resist material by measuring the transfer curves of graphene FETs. One reason for graphene contamination by resist is thought to be the $\pi-\pi$ stacking of the aromatic rings contained in novolac resist to graphene. Decreasing the number of novolac resist processes on graphene was effective in suppressing the graphene deterioration. Acryl resist, the base resin of which does not contain aromatic rings, also suppressed the graphene deterioration compared with novolac resist, the base resin of which contains benzene rings. The PAG that has a steric hindrance from approaching a graphene sheet improved the transfer characteristics of the FETs compared with the PAG without the 
steric hindrance. By taking these measures, the graphene deterioration was suppressed and the transfer characteristics of graphene FETs were improved.

\section{Acknowledgement}

We acknowledge support and useful discussion from Dr. Koji Asakawa and Dr. Naoko Kihara.

\section{References}

1. K. S. Novoselov, A. K. Geim, S. V. Morozov, D. Jiang, Y. Zhang, S. V. Dubonos, I. V. Grigorieva, and A. A. Firsov, Science, 306 (2004) 666.

2. L. Liao, J. Bai, R. Cheng, Y. Lin, S. Jiang, Y. Qu, Y. Huang, and X. Duan, Nano Lett., 10 (2010) 3952.

3. N. Harada, K. Hayashi, M. Kataoka, J. Yamaguchi, M. Ohtomo, M. Ohfuchi, I. Soga, D. Kondo, T. Iwai, and S. Sato, IEDM 2016 proceedings, 16 (2016) 477.

4. F. Schedin, A. K. Geim, S. V. Morozov, E. W. Hill, P. Blake, M. I. Katsnelson, and K. S. Novoselov, Nat. Material, 6 (2007) 862.

5. Y. Ohno, K. Maehashi, Y. Yamashiro, and K. Matsumoto, Nano Lett., 9 (2009) 3318.

6. M. Liu, X. Yin, D. Ulin-Avila, B. Geng, T. Zentgraf, L. Ju, F. Wang, and X. Zhang, Nature, 474 (2011) 64.

7. T. Mueller, F. Xia, and P. Avouris, Nature Photon., 4 (2010) 297.

8. X. Li, W. Cai, J. An, S. Kim, J. Nah, D. Yang, R. Piner, A. Velamakanni, I. Jung, E. Tutuc, S. K. Banerjee, L. Colombo, and R. S. Ruoff, Science, 324 (2009) 1312.

9. J. Lee, E. K. Lee, W. Joo, Y. Jang, B. Kim, J. Y. Lim, S. Choi, S. J. Ahn, J. R. Ahn, M. Park, C. Yang, B. L. Choi, S. Hwang, and D. Whang, Science, 344 (2014) 286.

10. C. Berger, Z. Song, X. Li, X. Wu, N. Brown, C. Naud, D. Mayou, T. Li, J. Hass, A. N. Marchenkov, E. H. Conrad, P. N. First, and W. A. de Heer, Science, 312 (2006) 1191.

11. Z. Cheng, Q. Zhou, C. Wang, Q. Li, C. Wang, and Y. Fang, Nano Lett., 11 (2011) 767.

12. Y. Dan, Y. Lu, N. J. Kybert, Z. Luo, and A. T. Charlie Johnson, Nano Lett., 9 (2009) 1472.

13. H. Ito, "Rejisuto zairyou (Resist material)", Kyoritsu Shuppan Co. Ltd., Tokyo (2005) p.13 (in Japanese).
14. Y. Kaimoto, K. Nozaki, S. Takeuchi, and N. Abe, Proc. SPIE, 1672 (1992) 66.

15. K. Nozaki, K. Watanabe, T. Namiki, M. Igarashi, Y. Kuramitsu, and E. Yano, Jpn. J. Appl. Phys., 35 (1996) L528.

16. K. Nozaki, K. Watanabe, E. Yano, A.Kotachi, S. Takechi, and I. Hanyu, J. Photopolym. Sci. Technol., 9 (1996) 509.

17. S. M. Sze, and K. K. Ng, "Physics of semiconductor devices", John Wiley \& Sons, Inc., Hoboken, (2007) p. 305.

18. M. J. Frisch, G. W. Trucks, H. B. Schlegel, G. E. Scuseria, M. A. Robb, J. R. Cheeseman, G. Scalmani, V. Barone, B. Mennumli, G. A. Petersson, H. Nakatsuji, M. Caricato, X. Li, H. P. Hratchian, A. F. Izmaylov, J. Bloino, G. Zheng, J. L. Sonnenberg, M. Hada, M. Ehara, K. Toyota, R. Fukuda, J. Hasegawa, M. Ishida, T. Nakajima, Y. Honda, O. Kitao, H. Nakai, T. Vreven, J. A. Montgomery, Jr., J. E. Peralta, F. Ogliaro, M. Bearpark, J. J. Heyd, E. Brothers, K. N. Kudin, V. N. Staroverov, R. Kobayashi, J. Normand, K. Raghavachari, A. Rendell, J. C. Burant, S. S. Iyengar, J. Tomasi, M. Cossi, N. Rega, J. M. Millam, M. Klene, J. E. Knox, J. B. Cross, V. Bakken, C. Adamo, J. Jaramillo, R. Gomperts, R. E. Stratmann, O. Yazyev, A. J. Austin, R. Cammi, C. Pomelli, J. W. Ochterski, R. L. Martin, K. Morokuma, V. G. Zakrzewski, G. A. Voth, P. Salvador, J. J. Dannenberg, S. Dapprich, A. D. Daniels, Ö. Farkas, J. B. Foresman, J. V. Ortiz, J. Cioslowski, and D. J. Fox, Gaussian 09, Revision E.01, Gaussian, Inc., Wallingford CT, (2013).

19. A.D. Becke, J. Chem. Phys., 98 (1993) 5648.

20. C. Lee, W. Yang, R.G. Parr, Phys. Rev. B, 37 (1988) 785.

21. W. Stevens, H. Basch, and J. Krauss, J. Chem. Phys., 81 (1984) 6026.

22. X. Wang, X. Li, L. Zhang, Y. Yoon, P. Weber, H. Wang, J. Guo, and H. Dai, Science, 324 (2009) 768.

23. J. Yan, Y. Zhang, P. Kim, and A. Pinczuk, Phy. Rev. Lett., 98 (2007) 166802.

24. A. C. Ferrari, J. C. Meyer, V. Scardaci, C. Casiraghi, M. Lazzeri, F. Mauri, S. Piscanec, D. Jiang, K. S. Novoselov, S. Roth, and A. K. Geim, Phys. Rev. Lett., 97 (2006) 187401. 